\title{
Causal feedforward control of a stochastically excited fuselage structure with active sidewall panel
}

\author{
Malte Misol, ${ }^{\text {a) }}$ Thomas Haase, and Hans Peter Monner \\ German Aerospace Center (DLR), Institute of Composite Structures and Adaptive Systems, \\ Braunschweig, Germany \\ Michael Sinapius ${ }^{\text {b) }}$ \\ Technical University Braunschweig, Institute of Adaptronics and Functional Integration, \\ Braunschweig, Germany
}

(Received 12 February 2014; revised 15 August 2014; accepted 3 September 2014)

\begin{abstract}
This paper provides experimental results of an aircraft-relevant double panel structure mounted in a sound transmission loss facility. The primary structure of the double panel system is excited either by a stochastic point force or by a diffuse sound field synthesized in the reverberation room of the transmission loss facility. The secondary structure, which is connected to the frames of the primary structure, is augmented by actuators and sensors implementing an active feedforward control system. Special emphasis is placed on the causality of the active feedforward control system and its implications on the disturbance rejection at the error sensors. The coherence of the sensor signals is analyzed for the two different disturbance excitations. Experimental results are presented regarding the causality, coherence, and disturbance rejection of the active feedforward control system. Furthermore, the sound transmission loss of the double panel system is evaluated for different configurations of the active system. A principal result of this work is the evidence that it is possible to strongly influence the transmission of stochastic disturbance sources through double panel configurations by means of an active feedforward control system.
\end{abstract}

(C) 2014 Acoustical Society of America. [http://dx.doi.org/10.1121/1.4895710]

PACS number(s): 43.40.Vn, 43.60.Cg, 43.55.Rg [SAF]

Pages: $1610-1618$

\section{INTRODUCTION}

The research work presented in this paper experimentally investigates the active feedforward control of an aircraft-relevant lightweight double panel structure. Although active vibration control (AVC) is considered, the results are of relevance for active structural acoustic control (ASAC) as well. This is due to the fact that both AVC and ASAC are based on the active control of structural vibration, though with regard to different performance metrics. It will be shown that the performance metric has no influence on causality since it is not part of the optimal feedforward control filter.

The activities are motivated by the fact that in the transportation sector, and especially in aerospace, the importance of lightweight construction is increasing while the requirements on sound transmission loss and acoustic comfort are retained. Fiber composite structures such as carbon fiber reinforced plastics (CFRP) are increasingly used in order to meet the requirements and regulations regarding the (energy) efficiency of vehicles. Unfortunately, the mechanical properties of CFRP structures (low mass density and high stiffness) result in low coincidence frequencies, high structural mobility, and efficient sound radiation (see, for example, Hambric and Fahnline ${ }^{1}$ ). As a result, exterior noise sources propagate

\footnotetext{
a) Author to whom correspondence should be addressed. Electronic-mail: malte.misol@dlr.de

b) Also at: German Aerospace Center (DLR), Institute of Composite Structures and Adaptive Systems, Braunschweig, Germany.
}

easily into the cabin, thus compromising the interior acoustics and the comfort of the passengers. In order to enhance their overall sound transmission loss, lightweight fuselage structures are typically constructed as double panel systems. Yet at low frequencies, the transmission loss of a double panel partition becomes even worse than that of a single panel partition of equal mass. This is especially true around the fundamental mass-air-mass resonance frequency

$$
f_{0}=\frac{1}{2 \pi}\left[\left(\frac{\rho_{0} c^{2}}{d}\right)\left(\frac{m_{1}+m_{2}}{m_{1} m_{2}}\right)\right]^{1 / 2},
$$

which is defined for an unbounded, uniform and nonflexible double-leaf partition according to Eq. (1) (see Fahy and Gardonio $^{2}$ ). In Eq. (1), the distance between the two inner surfaces of the double panel partition is described by $d$ and $m_{1}$ and $m_{2}$ are the masses per unit area of the two panels. Below this frequency, both panels are vibrating in phase, thus behaving like a single panel of mass per unit area $m=m_{1}+m_{2}$. By inspection of Eq. (1) it becomes clear that, as an increase of mass and volume (controlled by the panel distance $d$ ) is usually not an option, the application of conventional (passive) methods provide no satisfying solution to the problem of low-frequency sound transmission through lightweight structures. ASAC, however, offers a potentially lightweight-compliant solution since its efficiency and effectiveness is highest in the low-frequency range $(<500 \mathrm{~Hz})$. It involves the use of sensors and actuators connected by a suitable control law. 
The design of an ASAC system is a highly interdisciplinary task, which has been considered in theoretical, numerical, and experimental research work. Many publications deal with theoretical or numerical considerations of the design of ASAC systems for the reduction of sound transmission through double panel partitions. In many cases, the researchers focus on the investigation of different actuator concepts and methods applied to a double panel system. The issue of (spatial) coherence and causality, however, is rarely addressed. This manifests itself in the choice of a harmonic plane wave or a harmonic point force excitation, which does not impose constraints on causality and coherence.

Bao and Pan $^{3,4}$ analytically and experimentally investigate different active system configurations for the reduction of sound transmission through double panel partitions. A harmonic, plane acoustic wave is chosen for the disturbance excitation of the primary structure which, as already noted, excludes the effects of coherence and causality on the feedforward control performance. While in principle a plane wave excitation is well suited to approximate the source characteristics of shock-cell noise occurring in the aftsections of an aircraft (see, for example, Schiller ${ }^{5}$ or Montgomery ${ }^{6}$ ), it is not able to emulate the statistical properties of the turbulent boundary layer (TBL), which is another important factor for aft-cabin noise (see, for example, Schiller ${ }^{7}$ ). The publication of De Fonseca et al. ${ }^{8}$ considers the implementation of different actuator and sensor concepts applied to a double panel system. Here, too, a harmonic and spatially coherent disturbance excitation is chosen that excludes the effects of coherence and causality on the feedforward control performance. In Gardonio and Elliott, ${ }^{9}$ theoretical results are derived by using a mathematical model of an aircraft-relevant double panel system. Different actuator concepts are evaluated for the case of a spatially coherent harmonic disturbance excitation. All research work cited so far concludes that the so-called method of cavity control (which uses loudspeakers in the cavity as control actuators) is superior, since owing to the low modal density of the cavity it theoretically permits a broadband reduction of sound transmission through the double panel system. It is not discussed, though, whether a broadband reduction is achievable in the case of a stochastic disturbance source (for example, a TBL) and if the concept of cavity control would turn out to be superior under these circumstances as well. Furthermore, Carneal and Fuller ${ }^{10}$ conclude that the actuation of the secondary structure (the so-called panel control) is superior compared to the other methods (including cavity control). Still, since the experiments, as in the other cases, consider a harmonic, plane acoustic wave, the different result is presumably due to differences in the relative modal density of the involved subsystems (i.e., the panels and the fluid cavity). Sas et al. ${ }^{11}$ describe the theoretical and experimental investigation of a double panel system with active feedforward control. The disturbance excitation is realized by a single sound source driven either by a harmonic or by a bandlimited white noise signal. The reference signal of the active feedforward controller is taken from the noise generator, which improves the causality. This procedure, however, is not generally applicable in real aircraft operation since some of the external noise sources (for example, the TBL) are stochastic and the placement of (a sufficient number of) reference sensors outside the aircraft is impossible. Furthermore, as only one noise source is used in Sas et al., the spatial coherence will be equal to one, which again is not the case for a TBL. The spatial coherence of the pressures induced by a TBL is very low. A theoretical discussion based on the Corcos model ${ }^{12}$ can be found in Elliott et al. ${ }^{13}$ In order to implement a physically realizable active feedforward control system, however, the issues of (spatial) coherence, causality, and actuator feedback (on the reference sensors) need to be addressed. This paper addresses the issues of coherence and causality by exploiting the properties and the geometrical flexibility of a double panel system for the design of a physically realizable active feedforward control system.

In the light of the aforesaid, it can be stated that not much research work has been published with regard to the experimental realization and evaluation of actively controlled double panel structures in acoustic test facilities. Even fewer publications deal with the active structural acoustic control of aircraft-relevant double panel systems excited by broadband or stochastic disturbances. This is why the implications of the (spatial) coherence of the disturbance source and the causality of the feedforward control system on the disturbance rejection of the active system have not yet been sufficiently discussed. The present paper therefore provides experimental results of an aircraft-relevant double panel structure that is stochastically excited and whose secondary structure implements an active feedforward control system. Special emphasis is placed on the influence of coherence and causality on the feedforward control performance.

\section{THEORY}

\section{A. Active broadband feedforward control}

For reasons of clarity, the subsequent discussion of broadband feedforward control is limited to a single-input singleoutput (SISO) configuration. The theory of multiple-input multiple-output (MIMO) broadband feedforward control has been comprehensively discussed in Elliott ${ }^{14}$ or Kuo and Morgan. ${ }^{15}$

The basic scheme of a SISO feedforward control system is shown in Fig. 1. The primary path is denoted by $P$ and the secondary path by $S$. $P$ contains all dynamics and delays between the reference signal $x$ and the disturbance signal $d$. In a double panel system configuration, $x$ could be measured on the primary panel, which is subjected to the disturbance excitation, and $d$ could be measured on the secondary panel, which vibrates and radiates sound. $S$ contains all dynamics and delays between the low-pass-filtered (block $F$ ) output of the control filter $W$ and the compensation signal $y$. For the considered double panel system, $S$ includes the dynamics of the power amplifiers, the actuators and the double panel system itself. The difference between $d$ and $y$ is the error signal generated by the error sensors. The signal propagation delays $\delta$ occurring in the analog filters $F\left(\delta_{F}\right)$, in the secondary path $S\left(\delta_{S}\right)$ and in the real-time digital signal processing (DSP) system $\left(\delta_{\mathrm{DSP}}\right)$, which implements the finite impulse response (FIR) control filter $W$, are highlighted. Under the assumption of linearity and time invariance, the subsystems $F, S$, and $W$ 


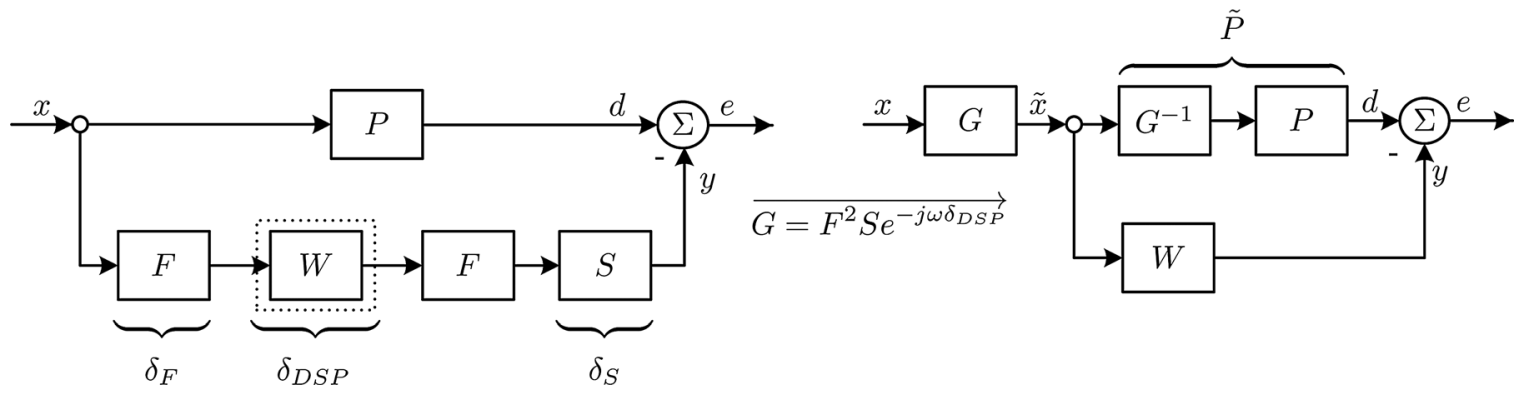

FIG. 1. Block diagram of a SISO feedforward control system with real delay $\delta$.

can be commuted and an augmented secondary path $G=F^{2} S e^{-j \omega \delta_{\mathrm{DSP}}}$ is defined, which includes all signal dynamics and propagation delays that are relevant to the active feedforward control system. The filtered reference signal $\tilde{x}$ results from filtering $x$ through $G$.

From Fig. 1, the optimal feedforward controller is given by

$$
W_{\mathrm{opt}}=\tilde{P}=\frac{P}{G} .
$$

A representation of the optimal feedforward controller in the discrete frequency domain

$$
W_{\mathrm{opt}}(k)=\frac{S_{\tilde{x} d}(k)}{S_{\tilde{x} \tilde{x}}(k)}
$$

in terms of the power spectral density (PSD) $S_{\tilde{x} \tilde{x}}$ of $\tilde{x}$ and the cross-power spectral density $S_{\tilde{x} d}$ of $\tilde{x}$ and $d$ is provided in Eq. (3). The parameter $k=0,1, \ldots, N-1$ describes the $k$ th frequency bin of the power spectral densities, which is associated with the discrete normalized frequency $\omega_{k}=2 \pi k / N$.

\section{B. Causality}

The physical realizability and the performance of a stochastically excited active feedforward control system crucially depend on causality. If the combination of primary path $P$ and augmented secondary path $G$, as shown in Fig. 1, is noncausal, then the optimal feedforward controller will be noncausal as well. Hence, for the practical implementation, the optimal feedforward controller must be mapped to its causal part. This, of course, generally implies a reduced control performance in terms of disturbance rejection. Yet it must be noted that, provided the reference signal itself is spectrally colored, a significant disturbance rejection might be achievable even if $P / G$ is noncausal. Owing to its importance, this section will further investigate the aspect of causality in relation to active feedforward control. As before, a SISO control system configuration will be applied.

Using Eq. (2), the (causally unrestricted) optimal feedforward controller in the continuous frequency domain is given by

$$
\begin{aligned}
W_{\mathrm{opt}}(\omega) & =\frac{P(\omega)}{G(\omega)}=\frac{|P(\omega)| e^{j \phi_{P}(\omega)}}{|G(\omega)| e^{j \phi_{G}(\omega)}} \\
& =\frac{|P(\omega)|}{|G(\omega)|} e^{j\left[\phi_{P}(\omega)-\phi_{G}(\omega)\right]} .
\end{aligned}
$$

It follows that the phase response of the optimal feedforward controller is given by $\Delta \phi=\phi_{P}-\phi_{G}$. Accordingly, the controller is physically realizable and causal if $\Delta \phi<0$ and noncausal if $\Delta \phi>0$. Regarding the control performance, however, there is a transition region around $\Delta \phi=0$. Since $\Delta \phi$ permits a quantification of the degree of (non)causality, its negative $-\Delta \phi=\phi_{G}-\phi_{P}$ will be termed causality margin. A positive causality margin is therefore associated with causality and a negative causality margin implies noncausality. According to Kong and Kuo, ${ }^{16}$ the degree of noncausality influences the controllable bandwidth of noise and the noise-cancelling efficiency decreases as the degree of noncausality increases. A quantitative prediction of the feedforward control performance under slight noncausality in the transition region is given in Lu et al. ${ }^{17}$ In Janocha and Liu ${ }^{18}$ it is shown that a sufficient (positive) causality margin is required in order to be able to accurately model the (delayed) inverse of a non-minimum-phase system (which will generally be the case for the systems under consideration).

If the causality margin is negative, though, the optimal causal SISO feedforward controller in the discrete frequency domain can be obtained by means of spectral factorization (see Elliott ${ }^{19}$ ). For this task, the cepstral method (see Oppenheim and Shafer ${ }^{20}$ ) is applied to $S_{\tilde{x} \tilde{x}}$. In the SISO case, the spectral factor $F$ of $S_{\tilde{x} \tilde{x}}$ is given by

$$
F(k)=\exp \left(D F T\left\{c(n) I D F T \ln \left[S_{\tilde{x} \tilde{x}}(k)\right]\right\}\right) .
$$

In Eq. (5), the discrete Fourier transform is denoted by DFT and its inverse by IDFT. The causality constraint on the cepstrum is realized by

$$
c(n)= \begin{cases}0, & \text { for } n<0 \\ 1 / 2, & \text { for } n=0 \\ 1, & \text { for } n>0\end{cases}
$$

thus eliminating the noncausal part. The different scaling of $c(n)$ between Elliott and Oppenheim and Shafer is due to the fact that the latter calculates the cepstrum of $|X|$ instead of $|X|^{2}$. According to Elliott, ${ }^{19}$ the optimal causal feedforward controller in the discrete frequency domain is given by

$$
W_{\text {optc }}(k)=\frac{1}{F(k)}\left\{\frac{S_{\tilde{x} d}(k)}{F^{*}(k)}\right\}_{+} .
$$

In Eq. (7), the complex conjugation is denoted by $*$ and $\{\cdot\}_{+}$represents the causal part of $\{\cdot\}$, which is calculated by transforming the argument of $\{\cdot\}$ to the discrete time domain (via the IDFT), setting the noncausal part of the impulse 
response to zero and transforming its causal part back to the discrete frequency domain (via the DFT). It is guaranteed that the resulting discrete filter equals its continuous counterpart at each frequency bin, provided that the causal part of the argument of $\{\cdot\}$ has a duration of less than $N / 2$ samples $\left(\right.$ see Elliott $\left.{ }^{19}\right)$. The optimal causal feedforward controller $W_{\text {optc }}$ described in Eq. (7) will be used for the evaluation of the influence of causality on the disturbance rejection in Sec. III B. It must be noted that Eqs. (7) and (3) are identical if the causality constraint $\{\cdot\}_{+}$is removed, because $S_{\tilde{x} \tilde{x}}=F F^{*}$.

In the general case of a MIMO system-as considered in Sec. III D-the calculation of the optimal causal feedforward controller could be accomplished by a MIMO version of Eq. (7), which is provided by Elliott. ${ }^{21}$ Unfortunately, the derivation of the spectral factor matrix $\mathbf{F}$ of $\mathbf{S}_{\tilde{x} \tilde{x}}$ is more difficult than the cepstral method described in Eq. (5) (which is only valid for a SISO system). Davis ${ }^{22}$ proposes a method for the spectral factorization of rational matrices of stable Laplace transforms and verifies his approach by means of a $2 \mathrm{I} 2 \mathrm{O}$ system $(2 \times 2$ matrix $)$. An alternative solution process for the calculation of the spectral factor in the MIMO case is given by Cook and Elliott. ${ }^{23}$ In contrast to the method of Davis, ${ }^{22}$ only a discrete frequency version of the PSD matrix is required here. Alternatively, the derivation of the optimal causal feedforward controller can be done in the time domain. This approach, which is described in Elliott, ${ }^{24}$ is used in this work. It is based on a matrix formulation of the error vector, which permits an explicit expression of the vector of optimal filter coefficients. The benefits of this method are its inherent causality, the specification of the number of filter weights and the possibility to include control effort into the performance metric. The required correlation matrices can be calculated in the discrete frequency domain, which improves the numerical efficiency and permits the use of frequency-response-data models (FRD) instead of state-space models (SSM). Furthermore, FRD models are generally more easily obtained from measurement or simulation data than SSM. Since the autocorrelation matrix has a block-Toeplitz structure, it is highly redundant and iterative methods are available to solve for the optimal filter weights. ${ }^{25,26}$

\section{Coherence}

The coherence between the reference and the disturbance signals is another important factor of influence on the disturbance rejection of a feedforward control system. According to Kuo and Morgan, ${ }^{27}$ the complex coherence function

$$
\gamma_{\tilde{x} d}(\omega):=\frac{S_{\tilde{x} d}(\omega)}{\sqrt{S_{\tilde{x} \tilde{x}}(\omega) S_{d d}(\omega)}}
$$

of two wide-sense stationary random processes $\tilde{x}(n)$ and $d(n)$ is defined according to Eq. (8). ${ }^{28}$ From this follows the magnitude-squared coherence as

$$
C_{\tilde{x} d}:=\left|\gamma_{\tilde{x} d}(\omega)\right|^{2}=\frac{\left|S_{\tilde{x} d}(\omega)\right|^{2}}{S_{\tilde{x} \tilde{x}}(\omega) S_{d d}(\omega)} .
$$

In Fig. 1, the error signal $e$ is given by the difference of $d$ and $y=W \tilde{x}$. According to Kuo and Morgan, ${ }^{29}$ the dependence of the error signal's PSD $S_{e e}(\omega)$ $=E|D(\omega)-W(\omega) \tilde{X}(\omega)|^{2}$ (E represents the expectation operator) on the magnitude-squared coherence $C_{\tilde{x} d}$ is given by

$$
\begin{aligned}
S_{e e}(\omega)= & {\left[1-C_{\tilde{x} d}(\omega)\right] S_{d d}(\omega) } \\
& +\left|W(\omega)-\frac{S_{\tilde{x} d}(\omega)}{S_{\tilde{x} \tilde{x}}(\omega)}\right|^{2} S_{\tilde{x} \tilde{x}}(\omega) .
\end{aligned}
$$

Under ideal conditions, i.e., when the system is completely linear, the disturbances are fully captured by the reference sensors and the active feedforward control system is causal, the coherence becomes identically one and the controller $W$ equals the optimal controller of Eq. (3). According to Eq. (10), this drives the error signal's PSD to zero. Under real conditions, the coherence will never be identically one and the causality constraint might be violated. This leads to a control-performance degradation, which can be estimated by Eq. (10). In the case of multiple uncorrelated disturbances (for example, a TBL excitation), the acquisition of coherent and time-advanced reference signals is a difficult task. A reasonable reference sensor configuration for a stochastic acoustic excitation (for example, a diffuse sound field) can be derived from a principal component analysis. The singular-value decomposition (SVD) of the cross-power spectral density matrix $\mathbf{S}_{\tilde{\mathbf{x}} \tilde{\mathbf{x}}}$ of a multitude of potential reference signals $\tilde{\mathrm{x}}=\left[\tilde{x}_{1}, \tilde{x}_{2}, \ldots, \tilde{x}_{n}\right]$ provides an insight into the number of uncorrelated disturbances and gives a lower bound on the required number of reference signals. ${ }^{30}$ The theoretical number of independent disturbance sources in a diffuse sound field observed on a linear microphone array equals two per wavelength. ${ }^{13}$

\section{EXPERIMENT}

\section{A. Experimental setup}

The experiments are performed in a sound transmission loss facility with a reverberant sending room and a semianechoic receiving room. The general setup is depicted in Fig. 2.

The geometrical details of the primary structure and the installation of the double panel system in the wooden mounting frame are provided in Fig. 3 . The primary structure $\left(P_{1}\right)$ has a CFRP skin, four T-shape aluminum frames and 21 L-shape aluminum stringers. The radius is $2820 \mathrm{~mm}$, which is typical for a long-range aircraft. $P_{1}$ is connected with the wooden mounting frame via shock-mounts located in the corners at the positions of the brackets of $P_{1}$. The clearance of the mounting frame is $1700 \times 1310 \mathrm{~mm}^{2}$, leaving a small air gap between $P_{1}$ and the mounting frame. This gap is elastically closed with a thin silicone foil that has a negligible impact on the structural damping. The secondary structures $\left(P_{2}\right)$ are connected with the frames of $P_{1}$ via structural holders at the positions of the lining brackets [in Fig. 3(a) the upper lining brackets are hidden by the upper lid]. Unlike in real aircraft, the cavity between $P_{1}$ and $P_{2}$ is not filled with absorbent material and, furthermore, is laterally enclosed (baffled) by the ring frames and by stiffened aluminum lids mounted on the top and bottom. This rather generic double 


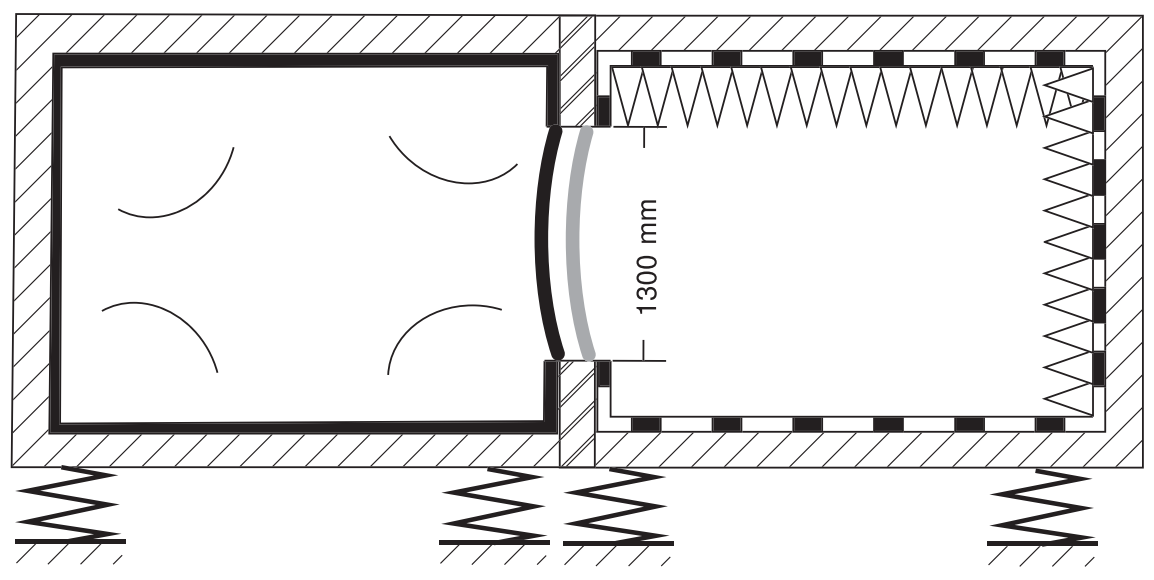

FIG. 2. Schematic view of the experimental setup in the transmission loss facility with the reverberant sending room to the left, the test specimen in the middle and the semi-anechoic receiving room to the right.

panel configuration is chosen in order to enhance the comparability of measurement data to the results of finite-element simulations. Original aircraft spacer linings are used for $P_{2}$, each consisting of a honeycomb core and top layers of fiberglass fabric. The lining located in the middle is augmented with ten inertial force actuators $A_{i}$ (mounted on the rear side) and 17 accelerometers, which are the error sensors $E_{i}$. Ten of the 17 accelerometers ( $E_{1}$ to $\left.E_{10}\right)$ are mounted opposite to the actuators [cf. Figure 4(b)]; this is considered-in the first approximation - to be a collocated, dual configuration. For reference sensing, ten accelerometers $R_{i}$ are mounted on $P_{1}$ at the positions indicated in Fig. 4(a). Table I provides an overview of the used hardware components and their settings. There, $f_{c}$ denotes the cutoff frequency of the low-pass filter, $F_{s}$ is the sampling frequency of the real-time system and $n_{\text {tap }}$ is the number of finite impulse response (FIR) filter weights.

As can be seen in Fig. 3(b), $P_{1}$ is directed to the reverberation room and $P_{2}$ points to the semi-anechoic room. Hence, $P_{1}$ is directly excited by the disturbance source and $P_{2}$ is indirectly excited by $P_{1}$ via the air cavity and the mechanical links. The force excitation of $P_{1}$ is realized by means of an electrodynamic exciter (shaker) mounted at the position indicated by the cross in Fig. 4(a). The diffusesound-field excitation of $P_{1}$ is synthesized in the reverberation chamber of the transmission loss facility by means of an omnidirectional dodecahedron sound source with shunted electrodynamic loudspeakers (all excited by the same signal). The excitation signal of the sound source is white noise bandlimited to the Nyquist frequency $F_{S} / 2$. The reverberation chamber has a volume of approximately $200 \mathrm{~m}^{3}$ and a mean reverberation time of approximately $5 \mathrm{~s}$ (averaged over third-octave bands from 80 to $5000 \mathrm{~Hz}$ ). It fulfills the ISO 3741 standard for frequencies above $100 \mathrm{~Hz}$.

\section{B. Causality}

The influence of causality on the disturbance rejection is theoretically discussed in Sec. II B. Figure 5 shows experimental results for a SISO system with a collocated, dual sensor-actuator pair $E_{2} / A_{2}$ on $P_{2}$ and three different reference sensors $R_{2}, R_{5}$, and $R_{8}$ mounted at different positions on $P_{1}$. A stochastic point force excitation is applied on $P_{1}$, collocated to reference sensor $R_{8}$ [cf. Fig. 4(a)]. It can be deduced from Fig. 5 that the disturbance rejection of the optimal causal feedforward controller $W_{\text {optc }}$ from Eq. (7) and the phase response of the optimal feedforward controller $W_{\text {opt }}$ from Eq. (3) are largely influenced by the distance between $R_{i}$ and $E_{2}$. Since the distance between $R_{8}$ and $E_{2}$ is largest, the disturbance rejection of $W_{\text {optc }}$ reaches the maximum in this configuration. This fact is reflected in the occurrence of the steepest phase response of $W_{\text {opt }}$ of all three configurations. It must be noted that in Fig. 5(b), only the phase response of the all-pass part of $W_{\mathrm{opt}}$ is shown. This is (a)

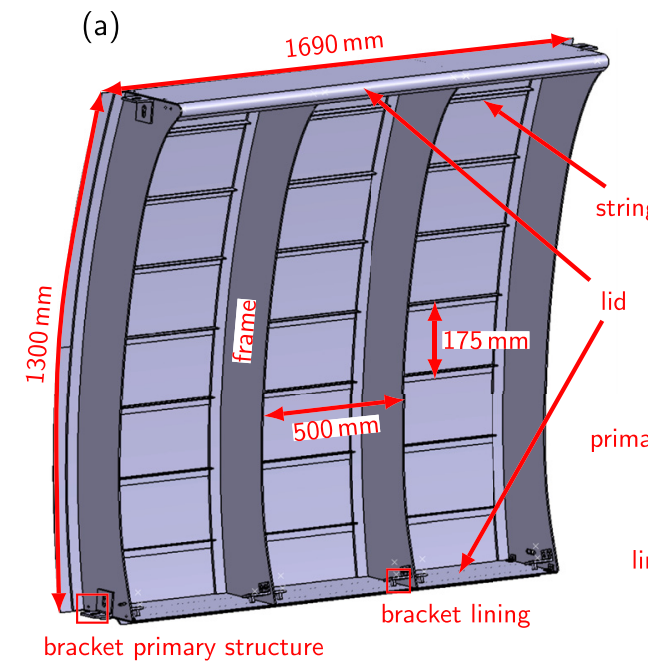

(b)

(b) 1 (reverberation room)

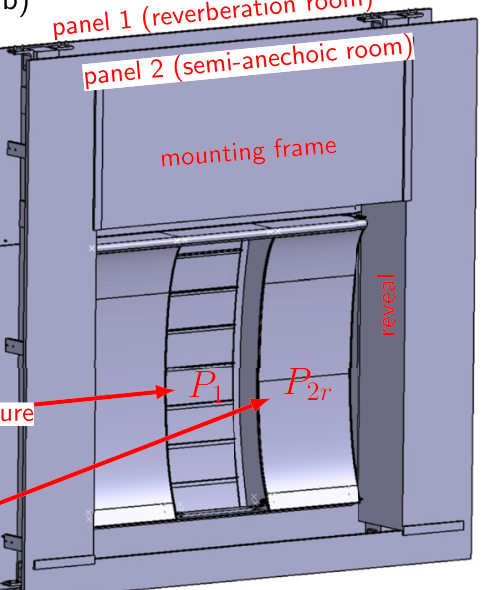

FIG. 3. (Color online) (a) Geometry of the primary CFRP panel and (b) installation of the double panel system in the mounting frame of the sound transmission loss facility. 

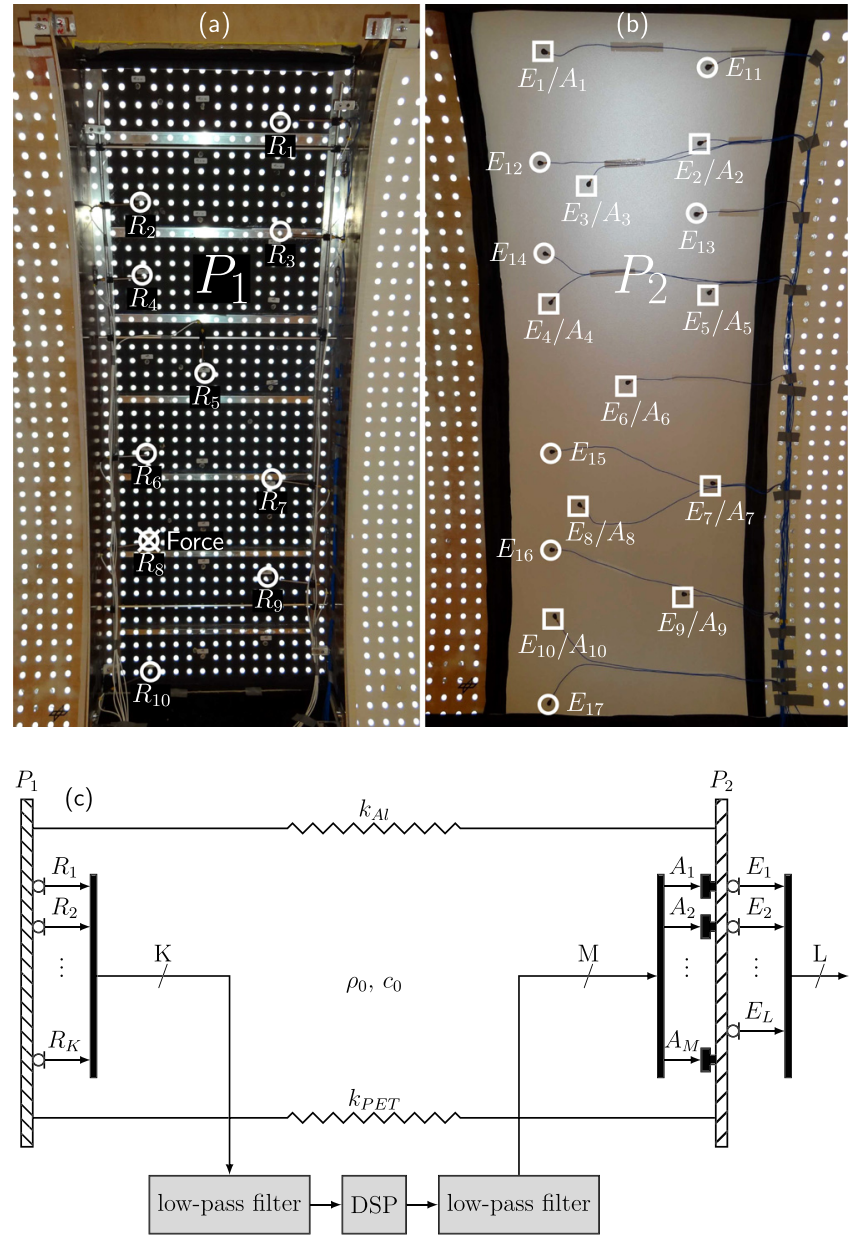

FIG. 4. (Color online) (a) Primary structure $\left(P_{1}\right)$ with reference sensors $\left(R_{i}\right)$ and force excitation point (Force), (b) sidewall panel $\left(P_{2}\right)$ with error sensors $\left(E_{i}\right)$ and actuators $\left(A_{i}\right)$, and (c) interconnection of actuators and sensors with the analog and digital signal processing (DSP).

justified by the fact that the phase response of the minimumphase part of $W_{\text {opt }}$ does not convey causality-relevant information and can therefore be omitted. In this sense, the negative phase response of $W_{\text {opt }}$ can be considered as an approximation of the causality margin of the optimal feedforward controller, which in turn leads to the conclusion that all three feedforward-control-system configurations are causal. This conclusion, however, is contradicted, or at least challenged, by the results depicted in Fig. 5(a), which show a significant disturbance rejection only for the configuration
TABLE I. Hardware components and settings of the AVC system.

\begin{tabular}{lcc}
\hline \hline Device & Type & Other \\
\hline Sensor & PCB 352A24 & $0.8(\mathrm{~g}), 10.2\left(\mathrm{mV} / \mathrm{m} / \mathrm{s}^{2}\right)$ \\
Actuator & Visaton EX45 & $60(\mathrm{~g})$ \\
Low-pass filter & Kemo & $f_{c}=480(\mathrm{~Hz}), 24(\mathrm{~dB} /$ Oct. $)$, \\
& CardMaster 255G & Gain 14 dB $(\times 5)$ \\
Real-time system & dSPACE DS1006 & $F_{s}=1000(\mathrm{~Hz})$ \\
Digital control filter & FIR & $n_{\text {tap }}=350$ \\
\hline \hline
\end{tabular}

with reference sensor $R_{8}$. A possible explanation for the limited disturbance rejection of the configurations with $R_{5}$ and $R_{2}$ is the observation of Janocha and Liu, ${ }^{18}$ mentioned in Sec. II B, that a sufficient (positive) causality margin is required in order to be able to accurately model the (delayed) inverse of a non-minimum-phase system. It can be concluded, then, that the causality margin is insufficient in the configurations with $R_{2}$ and $R_{5}$. Further insight might be gained from a closer inspection of the negative phase jumps introduced by the non-minimum-phase zeros and of the group delay $-d \phi / d \omega$ of $W_{\mathrm{opt}}$. These investigations, however, are beyond the scope of this paper and might be a topic of future research work.

\section{Coherence}

Figure 6 shows the magnitude-squared coherence function of the error sensor signal $E_{2}$ and the reference sensor signals $R_{2}, R_{5}$, or $R_{8}$ for the point force excitation and the magnitudesquared multiple coherence function of $E_{2}$ and either one $\left(R_{8}\right)$, five $\left(R_{1}, R_{4}, R_{7}, R_{8}, R_{10}\right)$ or all ten reference sensor signals (accelerometers on $P_{1}$ ) for the diffuse-sound-field excitation. According to Fig. 6(a), the coherence functions of $R_{2}, R_{5}$, or $R_{8}$ and $E_{2}$ are similar. Therefore, the first term in Eq. (10) is similar in all three cases. Nevertheless, the disturbance rejection shown in Fig. 5(a) differs between the configurations since the causality reflected in the second term of Eq. (10) is different. It appears that, the magnitude-squared coherence of $R_{2}$ and $E_{2}$ contains more noncausal parts than the magnitudesquared coherence of $R_{8}$ and $E_{2}$.

Figure 6(b) shows the multiple coherence function of $R_{i}$ and $E_{2}$ for the diffuse-sound-field excitation. The similarity between the coherence functions of the different reference sensor configurations is apparently lost. Two conclusions can be drawn from Fig. 6(b). (a)

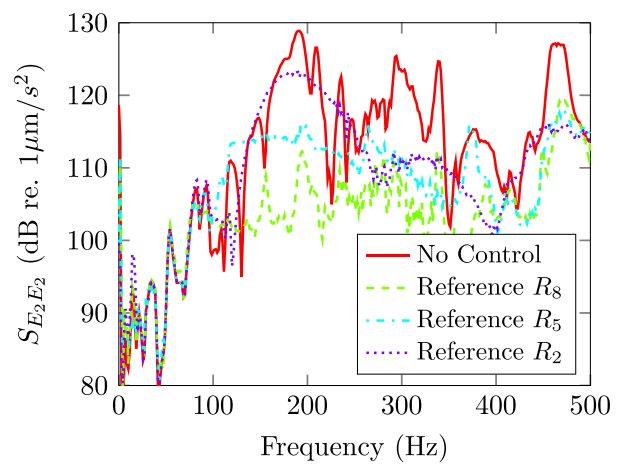

(b)

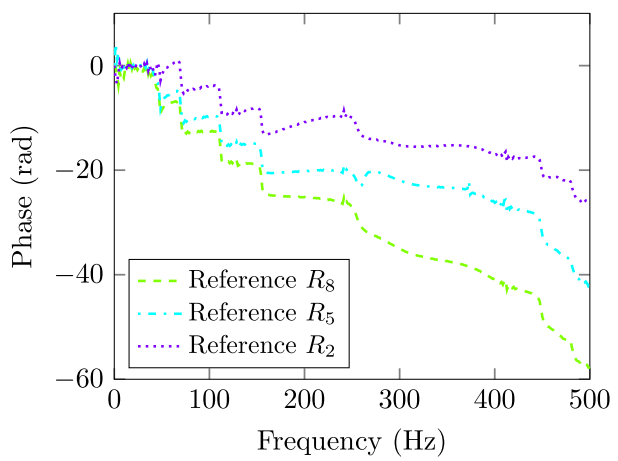

FIG. 5. (Color online) (a) Performance of the optimal causal feedforward controller and (b) phase response of the all-pass part of the optimal feedforward controller evaluated for three different reference sensors. 
(a)

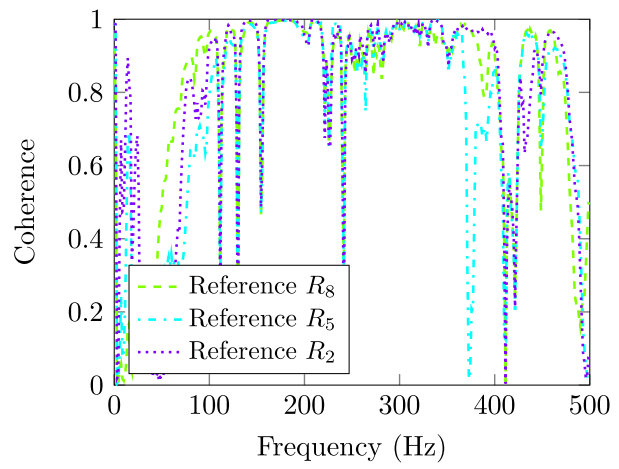

(b)

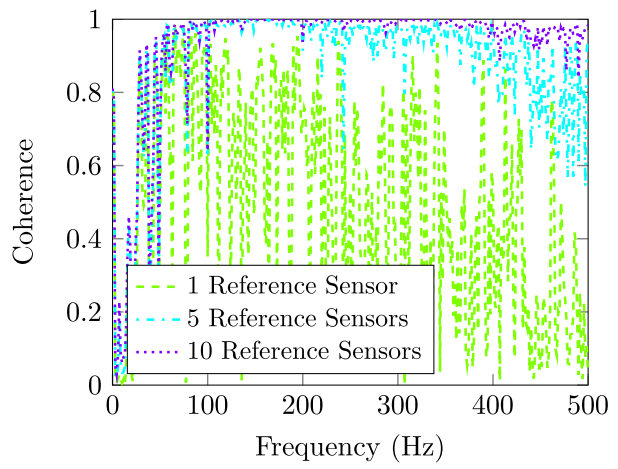

FIG. 6. (Color online) (a) Magnitudesquared coherence function for a point force and (b) a diffuse-sound-field excitation.
First, it can be stated that more than one reference sensor is required to achieve a high coherence ( $>90 \%)$, which is a somewhat surprising result since the diffuse sound field is excited by a single sound source. An explanation and a discussion of the spatial coherence of non-ideal diffuse sound fields and its implications on active feedforward control systems can be found in Misol et al. ${ }^{31}$ Then again the spatial coherence of the synthesized diffuse sound field largely exceeds that of an ideal diffuse sound field, which is characterized by two uncorrelated components per wavelength (observed on a linear microphone array). ${ }^{13}$

Second, in the case of five or ten reference signals, the coherence exceeds the one calculated for the point force excitation. So the spatial coherence of the structural vibration induced by the diffuse sound field is obviously sufficiently captured by five or ten reference sensors. In cases where the spatial coherence of the disturbance source is very low (for example, the pressure field induced by a TBL), the double panel configuration provides the flexibility to place microphones in the acoustic cavity between the two panels in order to use them as reference sensors. This might - at least at low frequencies - enhance the multiple coherence of reference and disturbance signals (for a given number of reference sensors), but it comes at the cost of a significant deterioration of robustness due to the actuator feedback on the reference sensors.

\section{Control performance}

The performance of the active feedforward control system is evaluated for the diffuse-sound-field excitation described in Sec. III A. The vibration reduction is evaluated in terms of the summed PSD of the error sensor signals and the sound power reduction is determined by means of a sound-intensity probe according to the ISO 9614-2 standard. The PSD have a resolution bandwidth of $1.43 \mathrm{~Hz}$. Two different control system configurations are evaluated: first, a square MIMO system with 10 collocated, dual actuatorerror-sensor pairs and, second, a rectangular system with 10 collocated, dual actuator-error-sensor pairs and seven additional noncollocated error sensors (further information on square and rectangular feedforward control systems can be found in Minkoff ${ }^{32}$ ).

It should be noted that the considered control system configurations implement AVC systems. Thus, the active system focuses on the vibration reduction at the error sensors and not on the radiated sound power. Nevertheless, since both AVC and ASAC rely on the active control of structural vibration, the experiments are relevant and the results are valid for ASAC systems, too. It is clear from Fig. 1 that a manipulation of the error signal will affect both $P$ and $G$ and hence cancels out in Eq. (2). Consequently, the causality of an active feedforward control system is not influenced by any postprocessing of the error signals (for example, a radiation filter in the case of ASAC). In this sense, the results of this work can be considered as a necessary precondition for a successful implementation of an ASAC system with causal feedforward control.

Figure 7 shows the predicted (from the time-domain MIMO control design) and measured vibration reduction and the sound power reduction of the square control system. Reductions in active sound power are associated with positive $\Delta$-values. From Fig. 7 (a) it can be concluded that the feedforward control system is able to achieve a significant broadband reduction of the error signal's PSD, which, (a)

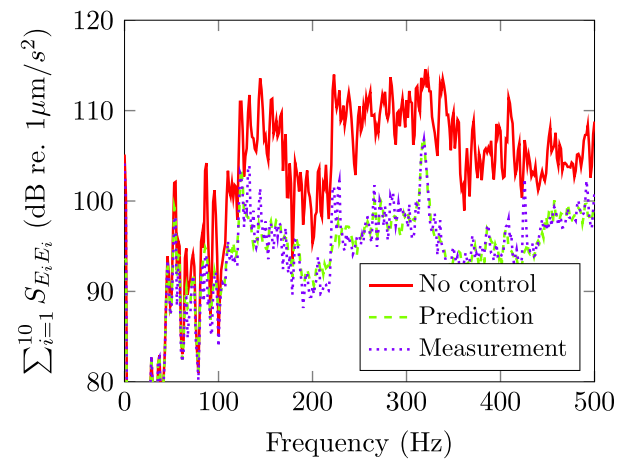

(b)

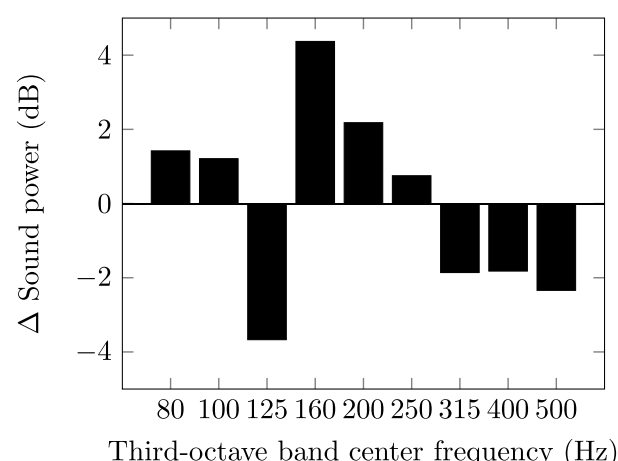

FIG. 7. (Color online) (a) Predicted and measured vibration reduction and (b) measured sound power reduction of the square MIMO feedforward control system with 10 reference sensors and 10 collocated actuator-error-sensor pairs according to Fig. 4. 
(a)

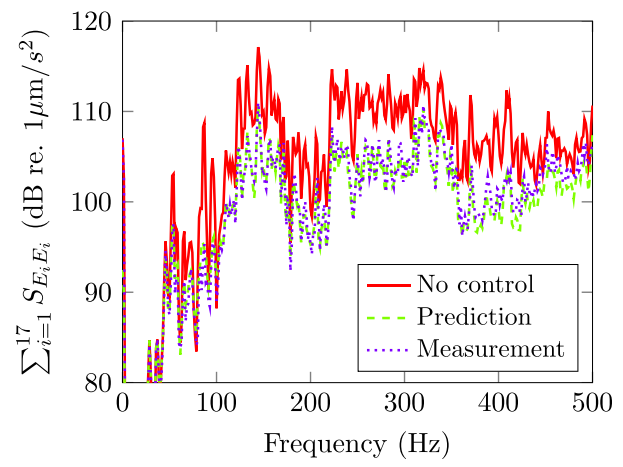

(b)

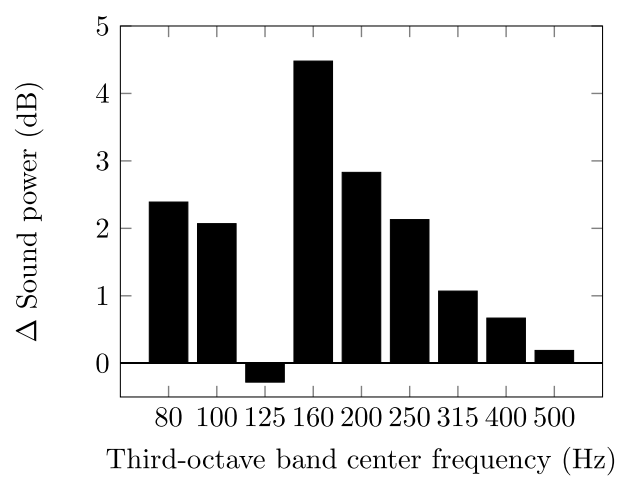

FIG. 8. (Color online) (a) Predicted and measured vibration reduction and (b) measured sound power reduction of the rectangular MIMO feedforward control system with 10 reference sensors, 10 collocated actuator-error-sensor pairs and seven noncollocated error sensors according to Fig. 4. according to Sec. III B, is only possible in a control configuration with sufficient causality margin. This is an important result as it proves the possibility to strongly influence the transmission of stochastic disturbance sources through double panel configurations by means of active feedforward control. Yet Fig. 7(b) shows that the definition of a local performance metric for the control design-as is the case here-will generally not lead to a reduction of transmitted sound power. Figure 8 shows the predicted (from the timedomain MIMO control design) and measured vibration reduction and the sound power reduction of the rectangular control system. Compared with the sound power reduction shown in Fig. 8(b), which is associated with a vibration reduction much less than that seen in Fig. 7(a), the acoustic performance of the square system shown in Fig. 7(b) is even worse. This behavior might, at least partially, be due to the so-called pinning effect described in Gardonio et al. ${ }^{33}$ Hence, in order to achieve higher reductions in transmitted sound power, a global, acoustically relevant performance metric must be formulated for the control design. Furthermore, a higher number of actuators might be required in order to achieve a strong reduction in transmitted sound power.

\section{CONCLUDING REMARKS}

The first part of this paper provides a theoretical discussion of active broadband feedforward control and its factors of influence coherence and causality. Section II gives a derivation of the causally unrestricted and restricted optimal feedforward controller for a SISO system configuration. Furthermore, in Sec. II B, the term causality margin is introduced and discussed with regard to its implications on the control performance.

The second part of this paper focuses on the experimental investigation of an aircraft-relevant double panel system equipped with an active sidewall panel. In Sec. III B, the disturbance rejection and the causality margin of a SISO feedforward control system is evaluated for a stochastic point force excitation of the primary structure of the double panel system. The experimental results show a clear relationship between the causality margin and the extent of the disturbance rejection of the active feedforward controller. This behavior has to be expected because of prior research work (see, for example, Janocha and $\mathrm{Liu}^{18}$ ) and could now be validated for a more complex vibro-acoustic system. Furthermore, Sec. III C considers the coherence between reference and disturbance signals for a point force and for a diffuse-sound-field excitation. It is shown that the spatial coherence of the structural vibration induced by the synthesized diffuse sound field falls below the one induced by the point force. Then again the spatial coherence of the synthesized diffuse sound field is artificially high compared to an ideal diffuse sound field, a condition which is mainly caused by the fact that a single sound source was used to synthesize the diffuse sound field in the reverberation chamber.

The main finding of this work is the evidence that it is possible to strongly influence the transmission of stochastic disturbance sources through double panel configurations by means of an active causal feedforward control system. This fact is considered as a necessary condition for the achievement of a significant reduction of sound power transmission through double panel systems. Future work will focus on the improvement of the acoustic performance by means of distributed actuation, sensing, and/or suitable filtering.

${ }^{1}$ S. A. Hambric and J. B. Fahnline, "Structural acoustics tutorial-Part 2: Sound-structure interaction," Acoust. Today 3(2), 9-27 (2007).

${ }^{2}$ F. Fahy and P. Gardonio, Sound and Structural Vibration: Radiation, Transmission and Response, 2nd ed. (Academic, Oxford/Burlington, 2007), p. 306.

${ }^{3}$ C. Bao and J. Pan, "Experimental study of different approaches for active control of sound transmission through double walls," J. Acoust. Soc. Am. 102(3), 1664-1670 (1997).

${ }^{4}$ J. Pan and C. Bao, "Analytical study of different approaches for active control of sound transmission through double walls," J. Acoust. Soc. Am. 103(4), 1916-1922 (1998).

${ }^{5}$ N. H. Schiller, "Decentralized control of sound radiation from periodically stiffened panels," Ph.D. thesis, Virginia Polytechnic Institute and State University, Blacksburg, Virginia, 2007, p. 17.

${ }^{6} \mathrm{~J}$. M. Montgomery, "Modeling of aircraft structural-acoustic response to complex sources using coupled fem-bem analyses," Collection of Technical Papers-10th AIAA/CEAS Aeroacoustics Conference (2004), Vol. 1, pp. 266-274.

${ }^{7}$ N. H. Schiller, "Decentralized control of sound radiation from periodically stiffened panels," Ph.D. thesis, Virginia Polytechnic Institute and State University, Blacksburg, Virginia, 2007, p. 115.

${ }^{8}$ P. De Fonseca, P. Sas, and H. Van Brussel, "Experimental study of the active sound transmission reduction through a double panel test section," Acta Acust. Acust. 85(4), 538-546 (1999).

${ }^{9} \mathrm{P}$. Gardonio and S. J. Elliott, "Active control of structure-borne and airborne sound transmission through double panel," J. Aircr. 36(6), 1023-1032 (1999). 
${ }^{10}$ J. P. Carneal and C. R. Fuller, “An analytical and experimental investigation of active structural acoustic control of noise transmission through double panel systems," J. Sound Vib. 272(3-5), 749-771 (2004).

${ }^{11}$ P. Sas, C. Bao, F. Augusztinovicz, and W. Desmet, "Active control of sound transmission through a double panel partition," J. Sound Vib. 180(4), 609-625 (1995).

${ }^{12}$ G. M. Corcos, "Resolution of pressure in turbulence," J. Acoust. Soc. Am. 35(2), 192-199 (1963).

${ }^{13}$ S. J. Elliott, C. Maury, and P. Gardonio, "The synthesis of spatially correlated random pressure fields,” J. Acoust. Soc. Am. 117(3), 1186-1201 (2005).

${ }^{14}$ S. J. Elliott, Signal Processing for Active Control (Academic, London, 2001), pp. 1-511.

${ }^{15}$ S. M. Kuo and D. Morgan, Active Noise Control Systems: Algorithms and DSP Implementations (Wiley, New York, 1996), pp. 1-408.

${ }^{16}$ X. Kong and S. M. Kuo, "Study of causality constraint on feedforward active noise control systems," IEEE Trans. Circuits Syst. II: Analog Digital Signal Process. 46(2), 183-186 (1999).

${ }^{17}$ J. Lu, H. Zou, and J. Tao, "Analysis of performance of feedforward active noise control systems in noncausality circumstances," Proc. of the Internoise 2012, CD-ROM (2012).

${ }^{18} \mathrm{H}$. Janocha and B. Liu, "Simulation approach and causality evaluation for an active noise control system," IEE Proc.-Control Theory Appl. 145(4), 423-426 (1998).

${ }^{19}$ S. J. Elliott, Signal Processing for Active Control (Academic, London, 2001), p. 69.

${ }^{20}$ A. V. Oppenheim and R. W. Shafer, Digital Signal Processing (Prentice Hall, Upper Saddle River, NJ, 1975), pp. 503ff.

${ }^{21}$ S. J. Elliott, Signal Processing for Active Control (Academic, London, 2001), p. 246
${ }^{22}$ M. Davis, "Factoring the spectral matrix," IEEE Trans. Autom. Control 8(4), 296-305 (1963).

${ }^{23}$ J. G. Cook and S. J. Elliott, "Connection between multichannel prediction error filter and spectral factorisation," IEE Electron. Lett. 35(15), 1218-1220 (1999).

${ }^{24}$ S. J. Elliott, Signal Processing for Active Control (Academic, London, 2001), pp. $237 \mathrm{ff}$.

${ }^{25}$ G. K. F. Rabl, "Recursive solution to wiener's multi-channel time filtering," Astron. Astrophys. 270, 552-556 (1993).

${ }^{26}$ E. A. Robinson, Multichannel Time Series Analysis with Digital Computer Programs, revised ed. (Holden-Day, San Francisco, 1978), pp. 1-454.

${ }^{27}$ S. M. Kuo and D. Morgan, Active Noise Control Systems: Algorithms and DSP Implementations (Wiley, New York, 1996), p. 56.

${ }^{28}$ G. C. Carter, "Coherence and time delay estimation," Proc. IEEE 75(2), 236-255 (1987).

${ }^{29}$ S. M. Kuo and D. Morgan, Active Noise Control Systems: Algorithms and DSP Implementations (Wiley, New York, 1996), p. 57.

${ }^{30}$ M. Akiho, M. Haseyama, and H. Kitajima, "Virtual reference signals for active noise cancellation system," J. Acoust. Soc. Jpn. (E) 19(2), 95-103 (1998).

${ }^{31}$ M. Misol, C. Bloch, H. P. Monner, and M. Sinapius, "Performance of active feedforward control systems in non-ideal, synthesized diffuse sound fields," J. Acoust. Soc. Am. 135(4), 1887-1897 (2014).

${ }^{32}$ J. Minkoff, "The operation of multichannel feedforward adaptive systems," IEEE Trans. Signal Process. 45(12), 2993-3005 (1997).

${ }^{33}$ P. Gardonio, E. Bianchi, and S. J. Elliott, "Smart panel with multiple decentralized units for the control of sound transmission. Part I: Theoretical predictions," J. Sound Vib. 274(1-2), 163-192 (2004). 